I N S T I T U T O

$\mathrm{DE}$

M E D I C I N A

T R O P I C A L

DE

S ÃO PAULO

JOURNAL OF THE SÃO PAULO INSTITUTE OF TROPICAL MEDICINE

${ }^{1}$ North Khorasan University of Medical Sciences, Vector-borne Diseases Research Center, Bojnurd, Iran

${ }^{2}$ Shahid Beheshti University of Medical Sciences, School of Medicine, Department of Medical Parasitology and Mycology, Tehran, Iran

${ }^{3}$ Razi Vaccine and Serum Research Institute, Mashhad, Iran

†These authors ( $\mathrm{AB}$ and $\mathrm{SS}$ ) contributed equally to this work

Correspondence to: Alireza Badirzadeh Shahid Beheshti University of Medical Sciences, School of Medicine, Department of Medical Parasitology and Mycology, Koodak-yar Ave., Daneshjoo Blvd, Evin, Chamran Highway, P.O. Box 19395-4719, Tehran, Iran

Tel: +982123872564

E-mail: badirzadeh@gmail.com

Sadaf Sabzevari

North Khorasan University of Medical

Sciences, Vector-borne Diseases Research Center, Taleghani Street.

P.O.Box 74877-94149, Bojnurd, Iran

Tel: +989369367632

E-mail: sadafsabzevari@gmail.com

Received: 17 July 2017

Accepted: 16 October 2017
http://dx.doi.org/10.1590/S1678-9946201860005

\section{First case report of atypical disseminated cutaneous leishmaniasis in an opium abuser in Iran}

\author{
Seyed Ahmad Hashemi ${ }^{1}$, Alireza Badirzadeh ${ }^{2, \dagger}$, Sadaf Sabzevari ${ }^{1 \dagger}$, Ali Nouri ${ }^{1}$, \\ Mohammad Seyyedin ${ }^{3}$
}

\section{ABSTRACT}

Leishmaniasis is a worldwide tropical infectious disease caused by different species of intracellular protozoa parasites of the genus Leishmania. Herein, we report a 78-year-old man with unusual diffuse cutaneous leishmaniasis (DCL) who had a history of opium abuse and chronic obstructive pulmonary disease (COPD). He had multiple papular, crusted and severely ulcerated lesions extended to his arm and chest. Direct smears and skin punch biopsy of the lesions were suggestive of leishmaniasis. Parasite DNA was amplified from ulcers, and identified as Leishmania major by PCR-RFLP, confirmed by sequencing analyses. The aim of the current study was to bring to attention this atypical form of disease in CL endemic countries. Thus, this is the first case of DCL in an opium abuser with COPD due to L. major in Northeastern Iran indicating that atypical and extensive forms of CL (DCL) owing to $L$. major are increasing in Iran.

KEYWORDS: DCL. Opium. L. major. Leishmaniasis. COPD. PCR-RFLP. Iran

\section{INTRODUCTION}

Leishmaniasis, as a cosmopolitan infectious disease, is caused by protozoa parasites from the Kinetoplastida Leishmania spp., which are transmitted by the female sandfly and affects almost 12 million people worldwide ${ }^{1,2}$. The infection outcome varies according to the Leishmania species from severe cutaneous lesions to erosive mucosal ulcers with severe dermal disfigurements, to a life threatening visceral disease, depending directly on the interaction between parasites and the host immunity ${ }^{3,4}$.

Iran is the main endemic focus of cutaneous leishmaniasis $(\mathrm{CL})^{5,6}$ in the Middle East and the main etiological agents of CL in the country are three species : L. major, $L$. tropica and less frequently $L$. infantum ${ }^{1,5,7,8}$. Different forms of CL have been reported in $\operatorname{Iran}^{1}$ including post kala-azar dermal leishmaniasis (PKDL) ${ }^{9}$, mucolymphatic leishmaniasis ${ }^{10,11}$ and recently diffuse cutaneous leishmaniasis (DCL) ${ }^{12}$. DCL, a rare and unusual form of CL, is characterized by the absence of cellular immunity, high number of parasites inside host cells, uncontrolled progression of disease and no response to antileishmanial therapy.

This is the first report of an atypical case of DCL caused by L. major in an opium abuser with chronic obstructive pulmonary disease (COPD) in the Northeastern region of Iran who had a good response to antileishmanial therapy.

\section{CASE REPORT}

A 78-year-old male patient was referred to the Imam Reza Hospital, North 
Khorasan University of Medical Sciences, Bojnurd, Iran, for evaluation of disseminated skin lesions over the arm and hemithorax. As shown in Figure 1, he presented with onemonth history of multiple skin lesions, which started with a small furuncle on the left forelimb which progressed to other parts of the arm and hemithorax. He was originally resident in Esfarayen city in the Northeastern region of Iran, which is one of the new endemic regions of cutaneous leishmaniasis (CL). The patient declared that prior to the dissemination of the lesion, he had edema and lymphedema over the arm and hemithorax. Physical examination was remarkable for mild peripheral edema and lymphedema, but in radiological and laboratory tests, there were no abnormalities. At the time of physical examination, mild peripheral edema and lymphedema, but no systemic symptoms such as fever, hepatosplenomegaly were observed; however, he had a 10-year history of opium smoking abuse, as well as Chronic Obstructive Pulmonary Disease (COPD) which had previously been treated with Salbutamol (100 $\mu \mathrm{g} / 4$ times (puffs) per day) and Fluticasone/Salmeterol Spray (250/50 $\mu \mathrm{g}$ twice a day). The patient was hospitalized with an initial suspicion of diffuse bacterial infection due to lymphedema, opium smoking and history of respiratory disease. Physical examination showed that he had multiple, disseminated and wet-looking skin lesions which were papular, crusted and severely ulcerated all over the left arm and hemithorax (Figure 1). Furthermore, the patient's lesions spread to his palm and all over the left finger nails which are very rare manifestations. It should be noted that three days before referring to the hospital, the patient bandaged up the lesions with plastic covers; therefore, all covered parts turned into creamy, wet-looking and severely inflammatory lesions which looked like second-degree burn wounds.

A hypothesis of leishmaniasis was made, and further

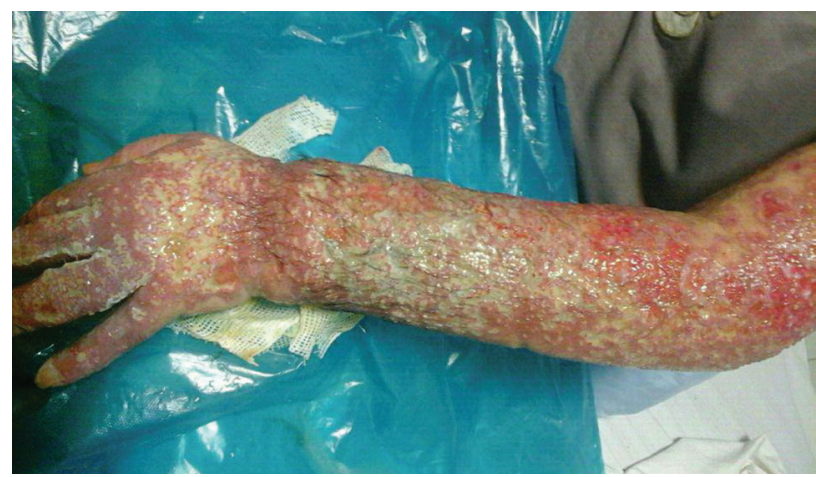

Figure 1 - Atypical disseminated cutaneous leishmaniasis (DCL) caused by Leishmania major in a patient with opium abuse and chronic obstructive pulmonary disease before antileishmanial therapy. The patient had multiple papular, crusted, severely ulcerated and creamy, wet-looking lesions that spread to his left arm and chest

evaluation by direct examination of the skin of the wrist and forearm using lesion scraping by a vaccinostyle, under sterile conditions was performed (Giemsa staining) revealing abundant intracellular Leishmania amastigotes inside and outside host cells (3+ parasitemia grade: 1-10 parasites/microscopic field) (Figure 2A). In addition, for histopathological examination, punch biopsy of lesions of the wrist and forearm were performed (Hematoxylin and Eosin staining: H\&E). Histopathological smears of wrist and forearm skin lesions revealed mild hyperkeratotic epidermis based on dermis infiltrated by numerous mixed inflammatory cells including abundant macrophages at dermis filled with amastigote forms of Leishmania (Figure 2B). Direct microscopy skin smears for acid-fast bacilli were negative. According to positive Giemsa-stained slide and skin biopsy, diagnosis of diffuse cutaneous leishmaniasis (DCL) was made Bone marrow aspiration showed no detectable Leishmania parasites among
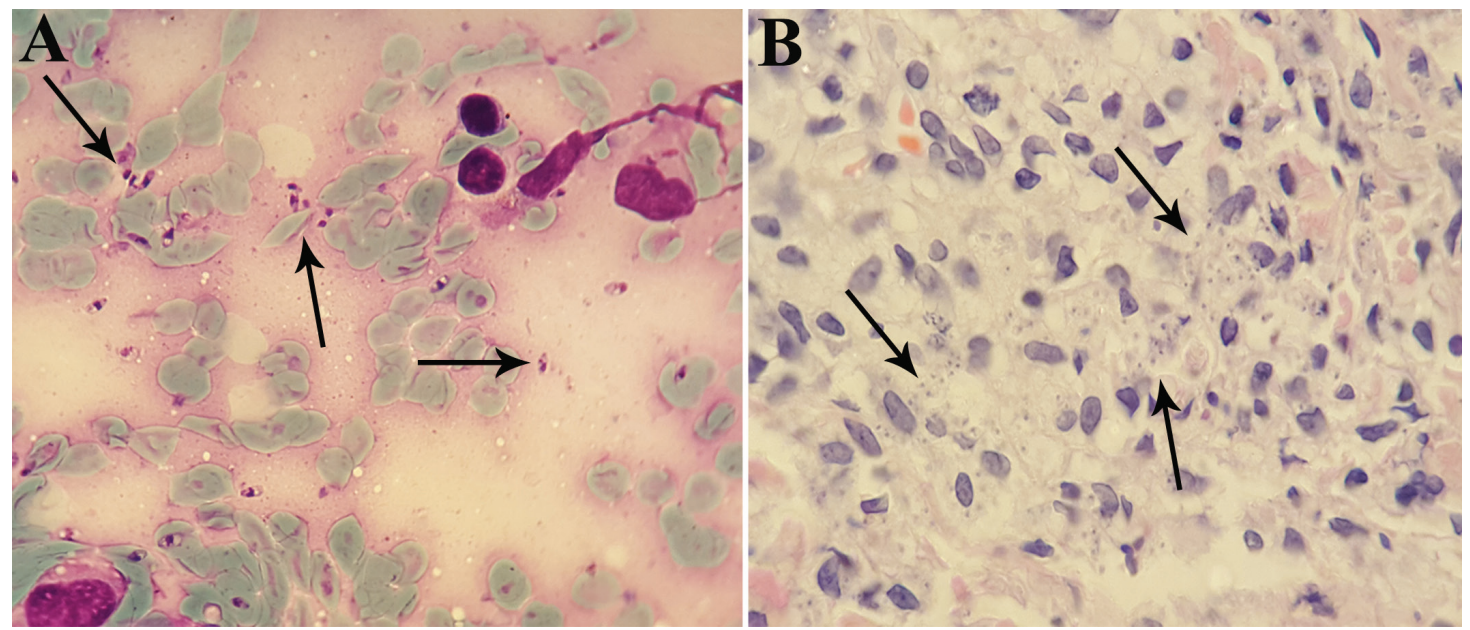

Figure 2 - A) Abundant intracellular amastigote forms of Leishmania major (arrows) in the Giemsa-stained smear; B) in the skin biopsy stained with Hematoxylin and Eosin, prepared from an ulcerated area of the left wrist and forearm of the patient with disseminated cutaneous lesions (original magnification: $\times 1,000$ ) 
myelogenous elements. All routine laboratory tests of the patient were normal. Viral markers (HIVAb, HBSAg, $\mathrm{HCVAb}$ ), and anti-Brucella Ab (Wright, Coombs Wright and 2-mercaptoethanol) were negative. In addition, serous fluid from the patient's skin lesion were cultured in Schneider's Drosophila medium (Sigma, Darmstadt, Germany), supplemented with 5\% heat-inactivated fetal bovine serum (HI-FBS) (Sigma, Germany). Isolated parasites were subcultured and monitored regularly to check promastigotes growth. In this experiment, $2 \times 10^{6}$ parasites $/ \mathrm{mL}$ at the logarithmic (2 days) growth phases were counted and used for DNA extraction.

Total genomic DNA of Leishmania parasites was extracted from cultured parasites and also from paraffinembedded blocks of the patient's skin biopsy using the GF-1 DNA extraction kit (GF-1, Vivantis, Canada), and Leishmania ITS1 was amplified by polymerase chain reaction (PCR). For identification of Leishmania species by PCR-RFLP, the PCR product was digested using HaeIII (BsuRI) enzyme ${ }^{5,13}$; L. major parasites were identified as the causative agents of DCL.

The patient's first PCR product was subjected to sequence analysis using the forward and reverse primers according to the manufacturer's instructions (Bioneer, South Korea). GenBank was searched for similar sequences and a significant similarity was found with other Leishmania species. The patient's ITS1 sequence was similar to the Iranian strain of L. major (AB04/HU64) (99\%).

The patient was initially treated with a bilayer wet dressing that was coated with silver sulfadiazine and petroleum salve/ointment along with vancomycin $(1 \mathrm{~g} / \mathrm{kg} / \mathrm{per}$ $12 \mathrm{~h})$ and ceftriaxone $(1 \mathrm{~g} / \mathrm{kg} / \mathrm{per} 12 \mathrm{~h})$ as broad spectrum antibiotics. At the same time, he received antileishmanial therapy including: meglumine antimoniate (Glucantime) $20 \mathrm{mg} / \mathrm{kg} /$ day for 10 days. Since he showed no significant improvement with Glucantime and developed pancytopenia as a complication of therapy, his treatment was discontinued at day 10. Consequently, he was placed on amphotericin B ( $3 \mathrm{mg} / \mathrm{kg} /$ per day for 10 days) and showed progressive and marked improvement of cutaneous lesions (Figure 3).

During hospitalization and successful treatment, he developed a severe lung infection and disseminated bronchiectasis probably due to his history of opium abuse and COPD; therefore, he was admitted to ICU and unfortunately died because of a systemic bacterial superinfection.

\section{DISCUSSION}

This is an unusual report of DCL caused by L. major in the Northeastern region of Iran in a patient with
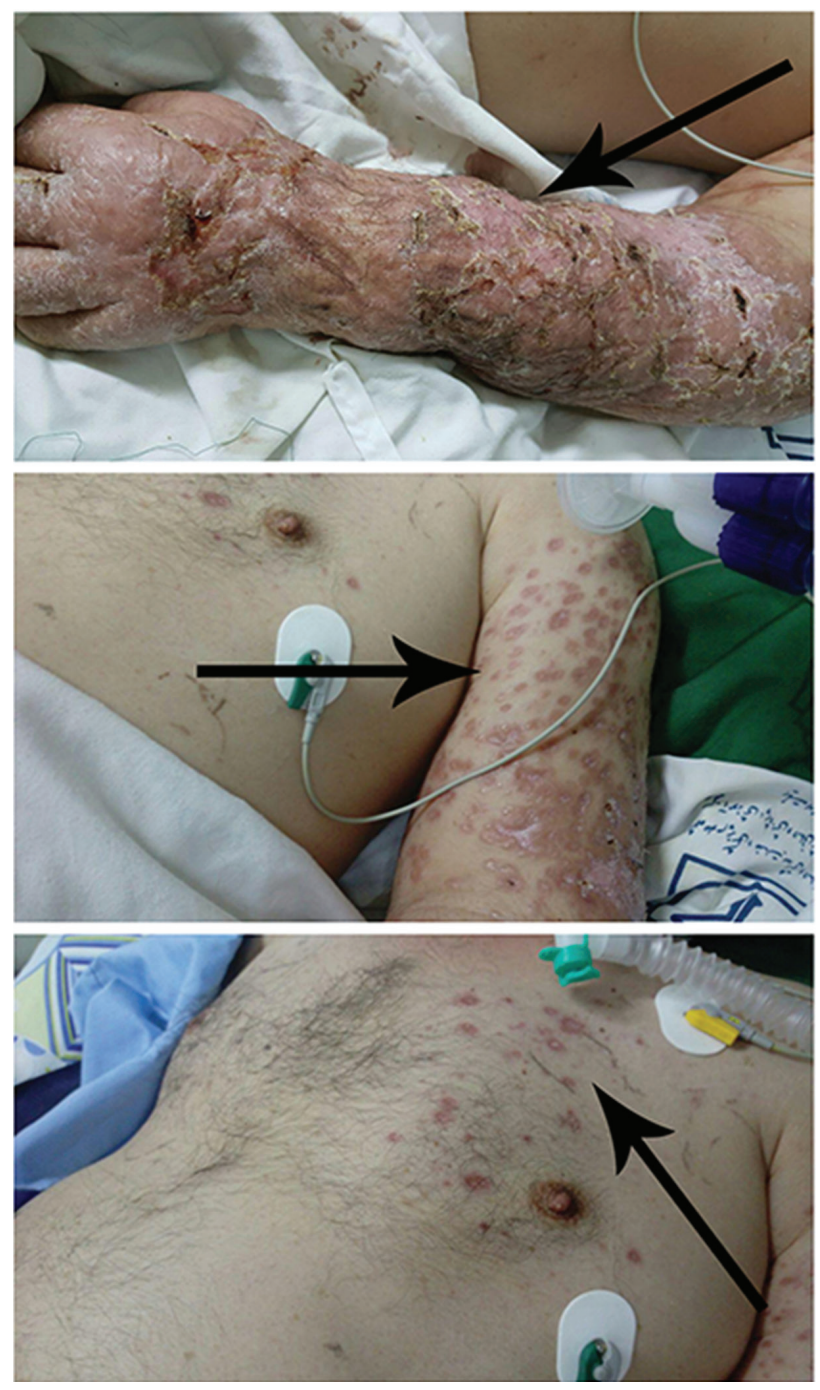

Figure 3 - Patient with atypical disseminated cutaneous leishmaniasis (DCL) showing good response to treatment with amphotericin B and regression of his cutaneous lesions

10 years history of opium abuse and respiratory disorder. He presented with an atypical form of CL with multiple, disseminated and creamy wet-looking skin lesions, which were papular, crusted and severally ulcerated all over the left arm and hemithorax.

DCL has been described in many countries, with a significant increase in the number of severe cases in highly endemic areas in the New World, and more recently in the Old World. It is characterized by an initial injury of the superficial and then the deep dermis followed by a high number of cutaneous polymorphic lesions with distinct features in the patient's body surface, with or without mucosal involvement and usually having no effective response to treatment ${ }^{14,15}$.

In the current case, the patient who was suffering from severe DCL described the initial appearance of a single furuncle exactly on the sand fly bite site of his left hand. 
Few months later, due to opium abuse and COPD, the patient's immune system was reduced; therefore, parasites could disseminate to other parts and lesions spread to the entire remote surface of the left arm and hemithorax, turning into disfiguring and extensive lesions within a few weeks.

Pathogenesis of Leishmania infection depends directly upon the interaction between host cells and the Leishmania parasite involving immunological mechanisms ${ }^{16}$. Nevertheless, in DCL, situations are very different and the exact immunopathogenesis has not been well established so far. DCL has been characterized in several underlying conditions such as malignant diseases, organ transplantation, HIV positive patients, and importantly in opium abusers, due to diminished immune responses ${ }^{17,18}$. Some authors have shown that DCL is associated with a defective cellular immunity to Leishmania genus, parasites allowing their uncontrolled replication within host cells. In our DCL case, the patient was an opium smoking abuser which led to COPD; however, the association of opiates, infection, and their mechanism of action, are complex and multifactorial ${ }^{18}$. Opium as a narcotic drug is more prevalent in different parts of Iran, especially in Northeastern areas ${ }^{19}$. In chronic abusers of narcotics as in our case, the replication of T lymphocytes diminished significantly, so that exposure of host macrophages cells to opiates significantly reduced the production of distinct T-helper 1 (Th1) cytokines by a downregulation of their coding genes ${ }^{20}$. Briefly, the cell mediated immunity (Th1) responses deviate into humoral immunity (Th2) via the cAMP dependent pathway, which eventually blocks Th1 cytokines expression such as TNF- $\alpha$, IFN- $\gamma$, IL-10 and IL-5, raising Th 2 cytokines expression ${ }^{19,20}$. Therefore, lower expressions of the Th1 cytokines cause the dissemination of the Leishmania parasites ${ }^{14,21}$. Moreover, some studies showed that alkaloid opioids induce immunosuppression and depress the cell function of natural killer (NK) and phagocytic cells; therefore causing dissemination of parasites to other parts on body ${ }^{19,22}$.

Although in the current study, L. major was identified as the causative species of DCL which is the most frequent species associated with CL in Iran, it has been rarely reported as the causative agent of DCL in both, immunosuppressed and non-immunosuppressed patients. The most frequently described agents of DCL in the country are $L$. tropica and $L$. infantum ${ }^{17}$.

Most cases of the DCL in Iran were reported in immunocompromised individuals such as HIV/AIDS patients; however, rare cases of the disease were described in patients with no immunosuppressive disorders ${ }^{17}$. In a study in Iran, Hajjaran et al. ${ }^{12}$ reported four cases of DCL with no immunossupression history in their medical notes and they did not respond to antileishmanial drugs.
These authors suggested a potential role of Leishmania spp. genome characteristics for causing DCL in healthy patients. Therefore, we should not undervalue the potential of parasites genomes and their virulent hybrids in the dissemination of parasites to other parts of the body surfaces ${ }^{12}$. It seems that the number of DCL cases in patients with and without immunosuppression in Iran is rapidly increasing.

Although it may be considered that there several limitations in the current study, further investigation must be conducted in all endemic parts of Iran to follow up the patients with atypical forms of CL such as DCL, and the parasites should be sequenced to identify the Leishmania strains in the country.

\section{CONCLUSIONS}

Although there has been a rise in the number of DCL cases in recent decades in Iran, this is a rare case report of DCL in a patient with history of opium abuse and COPD, parameters that have been poorly described in the literature. The precise identification of DCL causative species by molecular techniques is vital for prevention, treatment and control of the disease and also for epidemiological studies. It is possible that some DCL cases have been missed as a result of the poor reporting system and lack of diagnostic facilities; therefore, it is highly suggested that health workers and physicians are educated to ensure correct diagnosis and management of atypical and rare CL forms in both immunosuppressed and non-immunosuppressed patients. Moreover, we strongly suggest the use of molecular techniques for identification of Leishmania spp. and the epidemiology of these parasites in all parts of the country and differentiation of DCL from other forms of cutaneous lesions.

\section{ACKNOWLEDGMENTS}

The authors are grateful to the clinical research development center of Imam Reza hospital, North Khorasan University of Medical Science for their contribution and assistance. This report received no specific grant from any funding agency in the public, commercial or non-profit sectors.

\section{ETHICAL APPROVAL}

The current case report was approved by the local Ethics Committee of North Khorasan University of Medical Sciences, Bojnurd, Iran and a written consent was obtained from the patient. 


\section{CONFLICT OF INTERESTS}

We declare that we have no conflict of interest in relation to this work.

\section{REFERENCES}

1. Azizi K, Askari MB, Kalantari M, Moemenbellah-Fard MD. Molecular detection of Leishmania parasites and host blood meal identification in wild sand flies from a new endemic rural region, south of Iran. Pathog Glob Health. 2016;110:303-9.

2. Membrive NA, Kazuma FJ, Silveira TG, Teixeira JJ, ReinholdCastro KR, Teodoro U. Disseminated cutaneous leishmaniasis caused by Leishmania braziliensis in Southern Brazil. Rev Inst Med Trop Sao Paulo. 2017;59e37.

3. Badirzadeh A, Taheri T, Taslimi Y, Abdossamadi Z, HeidariKharaji M, Gholami E, et al. Arginase activity in pathogenic and non-pathogenic species of Leishmania parasites. PLoS Negl Trop Dis. 2017;11:e005774.

4. Montakhab-Yeganeh H, Abdossamadi Z, Zahedifard F, Taslimi Y, Badirzadeh A, Saljoughian N, et al. Leishmania tarentolae expressing CXCL-10 as an efficient immunotherapy approach against L. major-infected BALB/c mice. Parasite Immunol. 2017;39:e12461.

5. Abedi-Astaneh F, Hajjaran H, Yaghoobi-Ershadi MR, Hanafi-

Bojd AA, Mohebali M, Shirzadi MR, et al. Risk mapping and situational analysis of cutaneous leishmaniasis in an endemic area of Central Iran: a GIS-based survey. PLoS One. 2016;11:e0161317.

6. Badirzadeh A, Taheri T, Abedi-Astaneh F, Taslimi Y, Abdossamadi Z, Montakhab-Yeganeh H, et al. Arginase activity of Leishmania isolated from patients with cutaneous leishmaniasis. Parasite Immunol. 2017;39:e12454.

7. Heidari-Kharaji M, Taheri T, Doroud D, Habibzadeh S, Badirzadeh A, Rafati S. Enhanced paromomycin efficacy by solid lipid nanoparticle formulation against Leishmania in mice model. Parasite Immunol. 2016;38:599-608.

8. Badirzadeh A, Mohebali M, Asadgol Z, Soong L, Zeinali M, Mokhayeri Y, et al. The burden of leishmaniasis in Iran, acquired from the global burden of disease during 1990-2010. Asian Pac J Trop Dis. 2017;7:513-8.

9. Badirzadeh A, Mohebali M, Ghasemian M, Amini H, Zarei Z, Akhoundi B, et al. Cutaneous and post kala-azar dermal leishmaniasis caused by Leishmania infantum in endemic areas of visceral leishmaniasis, northwestern Iran 2002-2011: a case series. Pathog Glob Health. 2013;107:194-7.
10. Yaghoobi R, Hoghooghi-Rad N. Mucosal leishmaniasis: report of three cases. Arch Iran Med. 2001;4:138-40.

11. Mortazavi H, Mohebali M, Taslimi Y, Sadeghipour P, Ansari M, Kamyab K, et al. Hoarseness as the presenting symptom of visceral leishmaniasis with muco-cutaneous lesions: a case report. Iran J Parasitol. 2015;10:296-300.

12. Hajjaran H, Mohebali M, Akhavan AA, Taheri A, Barikbin B, Soheila NS. Unusual presentation of disseminated cutaneous leishmaniasis due to Leishmania major: case reports of four Iranian patients. Asian Pac J Trop Med. 2013;6:333-6.

13. Kazemi-Rad E, Mohebali M, Hajjaran H, Rezaei S, Mamishi S. Diagnosis and characterization of Leishmania species in Giemsa-stained slides by PCR-RFLP. Iran J Public Health. 2008;37:54-60

14. Vélez ID, Jiménez A, Vásquez D, Robledo SM. Disseminated cutaneous leishmaniasis in Colombia: report of 27 Cases. Case Rep Dermatol. 2015;7:275-86.

15. Hashiguchi Y, Gomez EL, Kato H, Martini LR, Velez LN, Uezato H. Diffuse and disseminated cutaneous leishmaniasis: clinical cases experienced in Ecuador and a brief review. Trop Med Health. 2016;44:2.

16. Pedraza-Zamora CP, Delgado-Domínguez J, Zamora-Chimal J, Becker I. Th17 cells and neutrophils: close collaborators in chronic Leishmania mexicana infections leading to disease severity. Parasite Immunol. 2017;39:e12420.

17. Jafari S, Hajiabdolbaghi M, Mohebali M, Hajjaran H, Hashemian H. Disseminated leishmaniasis caused by Leishmania tropica in HIV-positive patients in the Islamic Republic of Iran. East Mediterr Health. 2010;16:340-3.

18. Risdahl JM, Khanna KV, Peterson PK, Molitor TW. Opiates and infection. J Neuroimmunol. 1998;83:4-18.

19. Ayatollahi-Mousavi SA, Asadikaram G, Nakhaee N, Izadi A, Keikha N. The effects of opium addiction on the immune system function in patients with fungal infection. Addict Health. 2016;8:218-26.

20. Wang Y, Wang X, Ye L, Li J, Song L, Fulambarkar N, et al. Morphine suppresses IFN signaling pathway and enhances AIDS virus infection. PLoS One. 2012;7:e31167.

21. Gomes AH, Martines RB, Kanamura CT, Barbo ML, Iglezias SDA, Lindoso JA, et al. American cutaneous leishmaniasis: in situ immune response of patients with recent and late lesions. Parasite Immunol. 2017;39:e12423.

22. Yeager MP, Yu CT, Campbell AS, Moschella M, Guyre PM. Effect of morphine and $\beta$-endorphin on human $F c$ receptor-dependent and natural killer cell functions. Clin Immunol Immunopathol. 1992;62:336-43. 